\title{
El sapo de caña o "Bufo" (Rhinella marina) en Florida"
}

\section{S. A. Johnson, A. Wilson, and Armando J. Ubeda²}

El sapo de caña (Rhinella marina), a veces conocido como el "bufo", sapo gigante o marino, es nativo del extremo sur de Texas, México, América Central y la zona tropical de la América del Sur, pero está establecido en Florida. Los sapos de caña se introdujeron inicialmente en Florida como un método de control biológico de plagas en la década de 1930. Se suponía que los sapos comieran escarabajos que amenazaran el cultivo de la caña de azúcar, pero la población introducida no sobrevivió. En la década de 1950, un importador de mascotas libero alrededor de 100 sapos de caña (tal vez por accidente o a propósito, nadie está seguro) en el aeropuerto de Miami, y hay otros incidentes documentados de liberaciones intencionales en el sur de La Florida. Desde entonces, los sapos de caña se han extendido por gran parte del sur y centro de Florida. A partir de 2017, se establecieron en gran parte del sur de la península hasta el norte de Tampa (Figura 1), y ha habido varios avistamientos aislados en el norte de Florida y uno en el sureste de Georgia. Al parecer, una pequeña población se estableció en Deland, condado de Volusia, y en el condado Bay existió una población que sobrevivió durante varios años cerca de la Ciudad de Panamá. Los sapos de caña todavía están disponibles a través del comercio de mascotas, y los avistamientos aislados en el norte de Florida probablemente son sapos que se escaparon o fueron liberados por sus dueños.

Los sapos de caña han invadido con éxito ecosistemas en muchas partes del mundo donde las personas los introdujeron intencionalmente. Fueron introducidos deliberadamente en Hawái, Puerto Rico y muchas otras islas

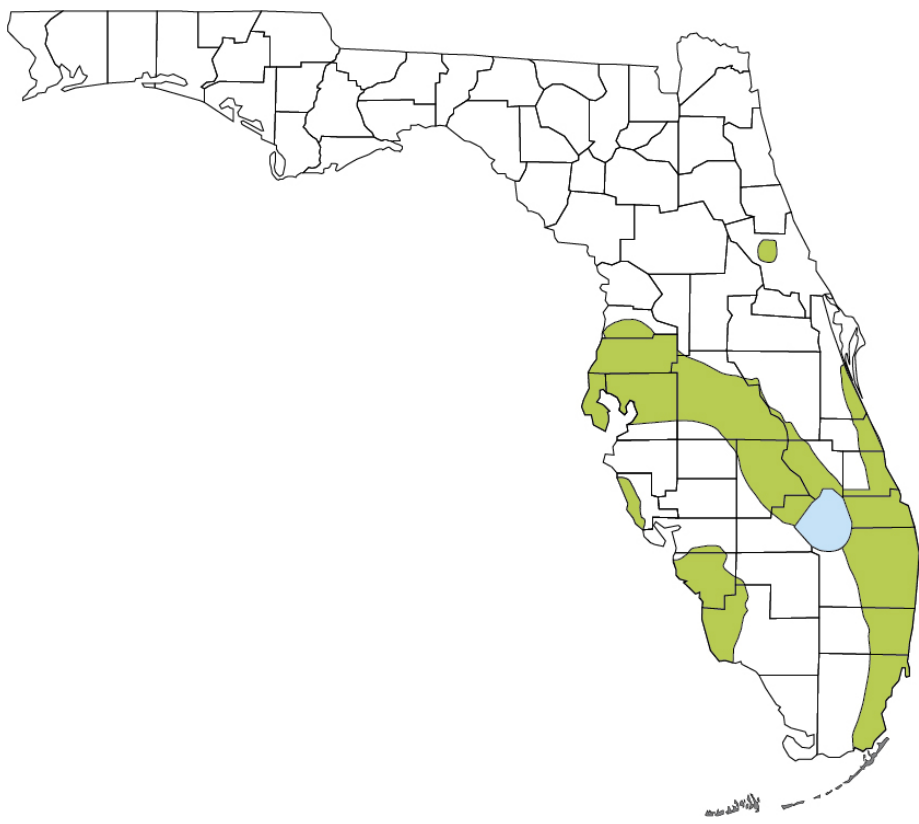

Figure 1. Distribución conocida de sapos de caña en Florida hasta enero de 2017. Obsérvese la población pequeña, aislada pero establecida en Deland, Condado de Volusia. [Haga clic en la figura para ampliarla]

del Caribe y el Pacífico en los siglos XIX y XX. Los sapos de caña son bien conocidos por su invasión en Australia, donde fueron llevados en la década de 1930 para controlar los escarabajos de caña, una plaga agrícola. Debido a su toxicidad, los sapos de caña han afectado severamente los ecosistemas australianos. Las tasas de mortalidad de muchos animales nativos de Australia son altas debido a la ingestión de sapos de caña (algunos ejemplos, lagarto monitor, cocodrilos de agua dulce, y numerosas especies

1. This document is WEC387, one of a series of the Department of Wildlife Ecology and Conservation, UF/IFAS Extension. Original publication date July 2017. Revised January 2020. Visit the EDIS website at https://edis.ifas.ufl.edu for the currently supported version of this publication.

2. S. A. Johnson, associate professor, Department of Wildlife Ecology and Conservation; A. Wilson, previous MS graduate student, Department of Wildlife Ecology and Conservation; and Armando J. Ubeda, UF/IFAS Extension Florida Sea Grant agent, Sarasota County; UF/IFAS Extension, Gainesville, FL 32611.

The Institute of Food and Agricultural Sciences (IFAS) is an Equal Opportunity Institution authorized to provide research, educational information and other services

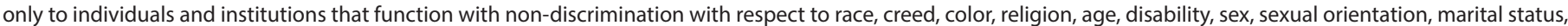

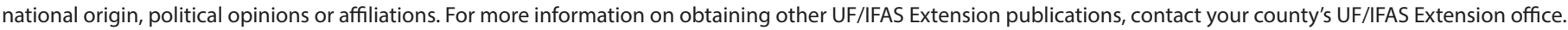
U.S. Department of Agriculture, UF/IFAS Extension Service, University of Florida, IFAS, Florida A \& M University Cooperative Extension Program, and Boards of County Commissioners Cooperating. Nick T. Place, dean for UF/IFAS Extension. 
de serpientes), y el número de depredadores nativos han disminuido en áreas donde los sapos tóxicos han invadido. Los sapos de caña continúan expandiéndose hacia el oeste a través del extremo superior de Australia hacia la región de Kimberly y hacia el sur en dirección a Sídney.

En su área de distribución nativa, los sapos de caña se encuentran en mayores densidades en hábitats modificados por humanos que en entornos naturales, siendo muy comunes en patios y áreas abiertas similares. Su capacidad para explotar el hábitat modificado por los humanos es un rasgo común entre muchas especies invasoras; los sapos de caña se han proliferado en patios, campos de golf, áreas agrícolas y tipos de hábitats similares en Florida. Afortunadamente, hay pocos registros de sapos de caña en los hábitats nativos de Florida, y no parecen invadir la mayoría de las áreas naturales del Estado. Aunque sus impactos ecológicos en Florida pueden estar limitados por el uso de su hábitat, los sapos de caña son una molestia significativa para las personas y potencialmente letales para las mascotas que atacan estos sapos de tierra.

\section{Identificación de los sapos de caña}

Los sapos de caña pertenecen a la familia científica Bufonidae. Los miembros de esta familia son conocidos como "sapos verdaderos", y en Florida hay cuatro especies nativas de bufónidos. El sapo del sur (Anaxyrus terrestris) y el sapo de roble (Anaxyrus quercicus) se analizan a continuación; el sapo de Fowler (Anaxyrus fowleri) y el sapo de la costa del Golfo (Incilius valliceps) solo se encuentran en el noroeste de Florida, en el occidente del área conocida como el Panhandle, y su rango de distribución no coincide con la de los sapos de caña, por lo tanto, estas dos especies nativas no se describen en este documento. Además de las cuatro especies de "sapos verdaderos" nativos de Florida, hay otra especie de sapo nativo, que posee las características generales de un sapo, pero que pertenece a una familia científica diferente: el sapo oriental pie de pala (Eastern spadefoot por su nombre en inglés). El sapo de caña puede confundirse con los sapos nativos, así que asegúrese de identificar correctamente la especie antes de tomar medidas para eliminar sapos invasores de su propiedad.

\section{¿Cómo sé si he encontrado un sapo de caña peligroso o un sapo nativo inofensivo?}

Primero, asegúrese de tener un sapo, luego para identificar la especie lea a continuación. Recuerde, todos los sapos son ranas, pero no todas las ranas son sapos. Los sapos son un tipo específico de rana.

\section{Características generales de los sapos:}

- Viven en el suelo y no son buenos trepadores

- Tienen cuerpos robustos con piernas cortas

- Tienen las patas traseras ligeramente palmeadas

- Tienen la piel seca y verrugosa

- Poseen glándulas venenosas (también llamadas glándulas parotoides) en los hombros

- Son moteados con varios tonos de gris, marrón, negro.

\section{Sapo de caña: invasivo}

- Pueden medir más de tres pulgadas de largo (las crías son más pequeñas)

- Glándulas venenosas agrandadas y casi triangulares, disminuyendo gradualmente, terminando en forma de punta (Figura 2)

- Sin pomos o crestas en la parte superior de la cabeza

- Crestas alrededor de los ojos y sobre la nariz

Los sapos de caña adultos miden entre tres y seis pulgadas de largo, y algunos individuos pueden llegar a medir ocho o nueve pulgadas. Los machos y las hembras se pueden distinguir por las diferencias en la coloración y la textura de la piel de la espalda; las hembras tienen la espalda lisa, moteada, marrón y blanca, mientras que la espalda rugosa de los machos es más amarilla (Figura 3). Los sapos de caña bebé recientemente transformados de renacuajos son del tamaño de las pasas y se confunden fácilmente con los sapos nativos.

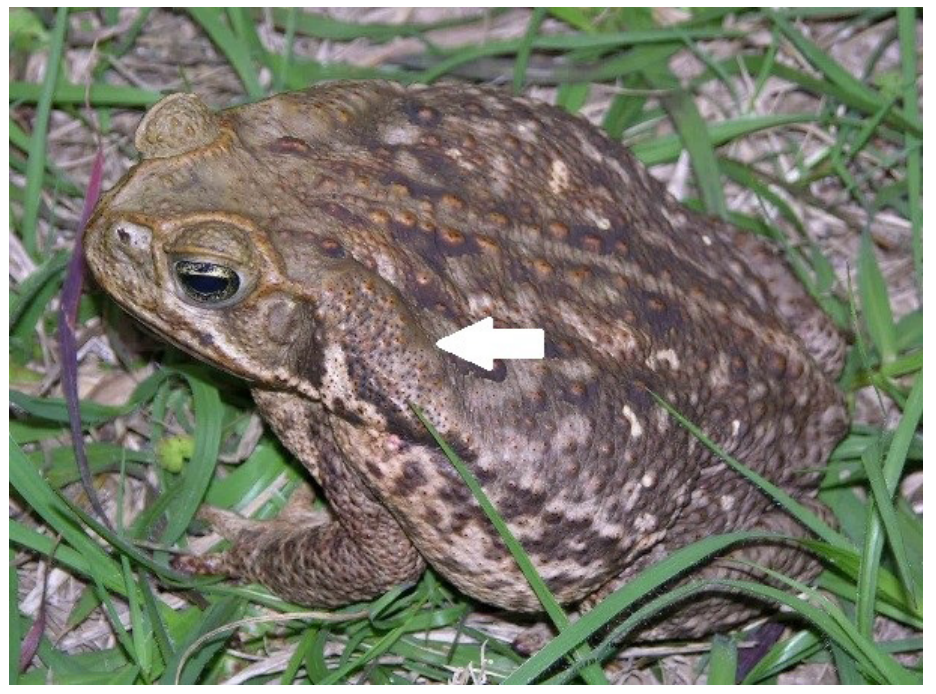

Figure 2. Los sapos invasores de caña tienen glándulas venenosas muy grandes en sus hombros (indicadas con una flecha); estas glándulas son algo triangulares, disminuyendo gradualmente hasta formar una punta. También tienen una cresta alrededor de los ojos y sobre la nariz.

Credits: Steve A. Johnson, UF/IFAS 


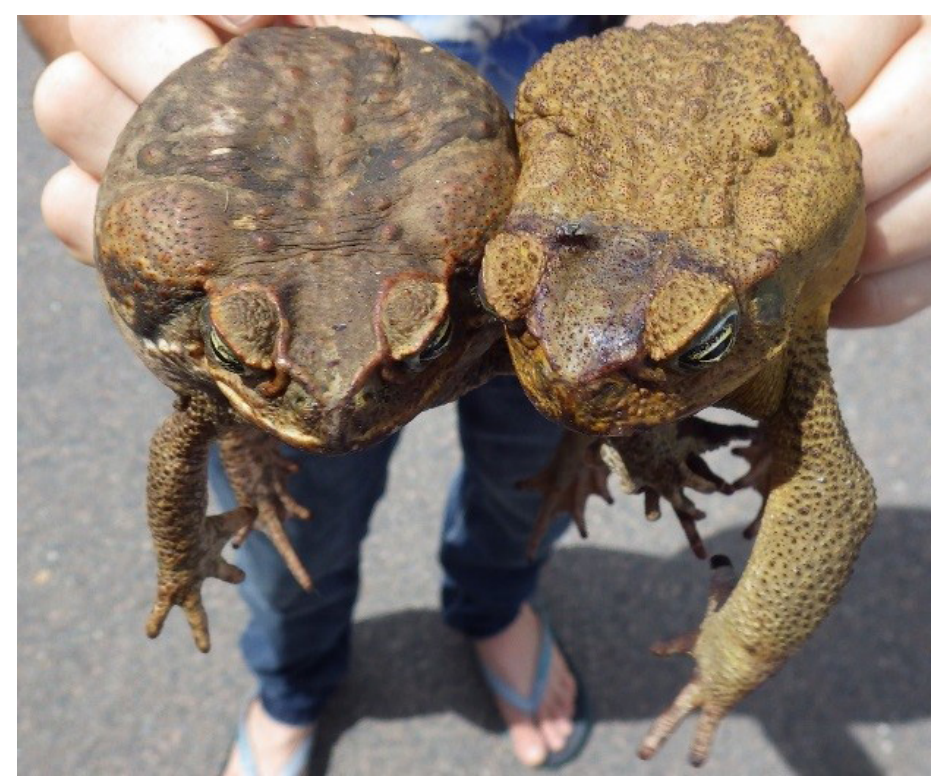

Figure 3. Sapo de caña hembra a la izquierda y macho a la derecha. Observe las diferencias en el color y la textura de la piel.

Credits: Steve A. Johnson, UF/IFAS

\section{Sapo del sur: nativo}

- Raramente tienen un tamaño de más de 3 pulgadas

- Las glándulas venenosas son pequeñas y ovaladas; no representan peligro para las mascotas

- Dos crestas visibles en la parte superior de la cabeza (las crestas no son obvias en los sapos del sur de menos de $\sim 1$ pulgada.)

- Cuerpo gris, marrón o rojizo.

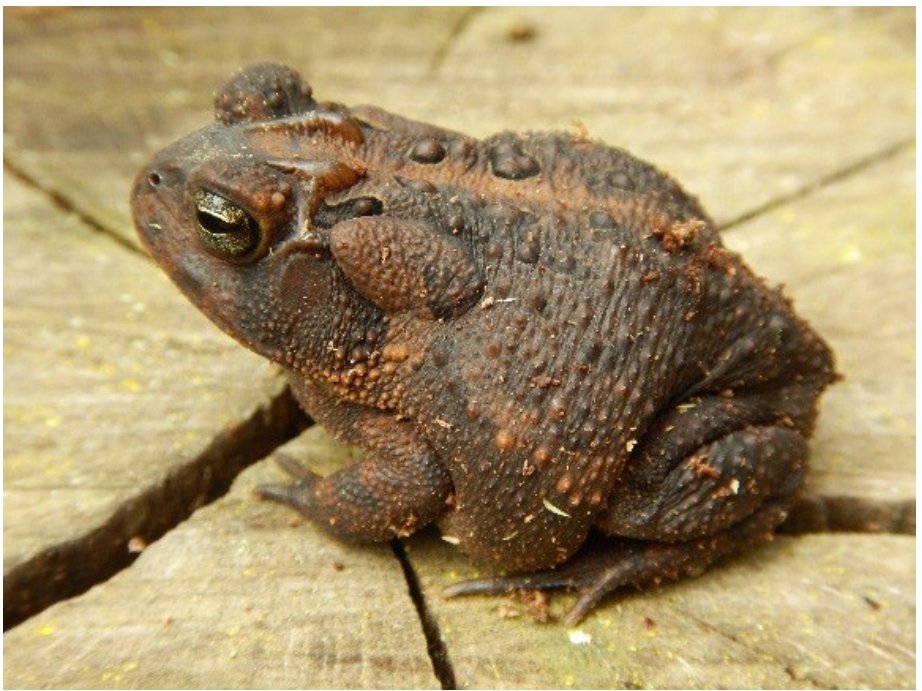

Figure 4. Los sapos del sur nativos tienen glándulas pequeñas y ovales en sus hombros y un par de crestas o crestas elevadas en la parte superior de sus cabezas.

Credits: Steve A. Johnson, UF/IFAS

\section{Sapo de roble: nativo}

- Nunca más grande que 1.5 pulgadas: es el sapo más pequeño de La Florida

- Las glándulas venenosas son pequeñas y ovaladas; no representan peligro para las mascotas

- Crestas indistintas en la parte superior de la cabeza

- Línea de color claro en el centro de la espalda

- Parte inferior de los pies de color naranja

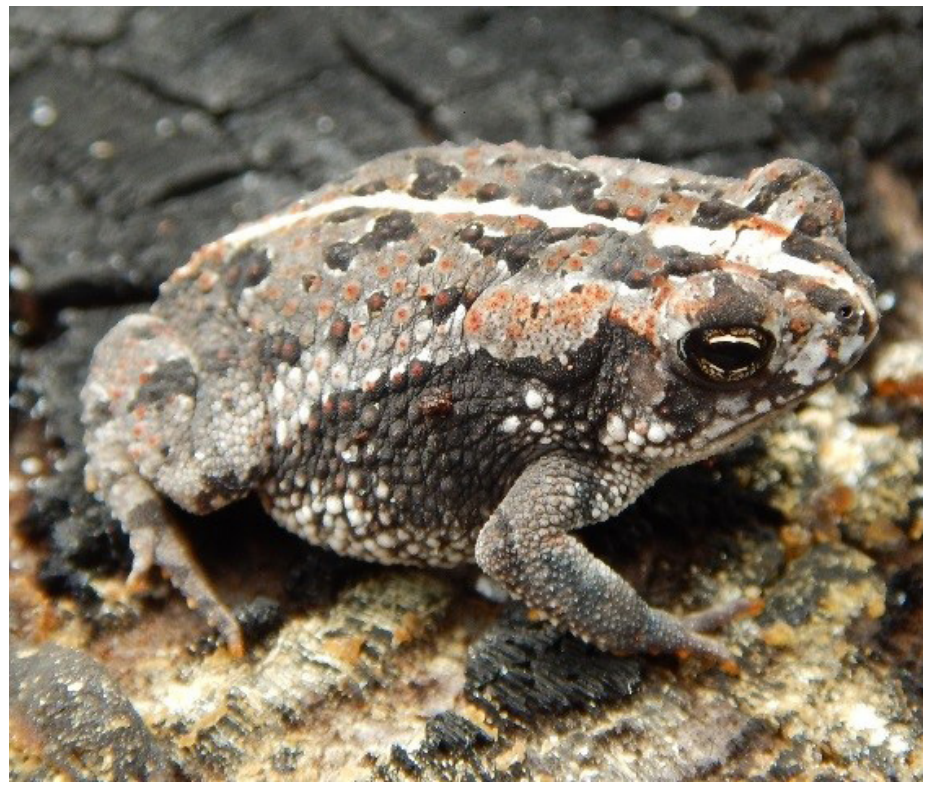

Figure 5. Los sapos de roble nativo son pequeños y tienen glándulas pequeñas y ovales en sus hombros. Las crestas emparejadas en la parte superior de sus cabezas son indistintas.

Credits: Steve A. Johnson, UF/IFAS

\section{Sapo oriental pie de pala: nativo}

- Raramente tienen un tamaño de más grande de dos pulgadas

- Glándulas venenosas aplanadas e indistintas; no representan un peligro para las mascotas

- Sin crestas en la parte superior de la cabeza

- A menudo tiene forma de reloj de arena y numerosas marcas amarillas en la espalda

- Una pronunciada espuela o "pala" de excavación en las patas traseras

Vea el video para obtener más información sobre cómo identificar con precisión los sapos en La Florida: https:// www.youtube.com/watch? $\mathrm{v}=\mathrm{RMzLes} 3 \mathrm{BaCo} \& \mathrm{t}=529 \mathrm{~s}$. 


\section{Ecología del sapo de caña}

Los sapos de caña son altamente adaptables y se encuentran en una variedad de tipos de hábitat. En su área de distribución nativa, viven en todas partes, desde la sabana hasta el bosque abierto, pero la vegetación densa parece actuar como una barrera para su movimiento. En Florida son particularmente comunes en patios, campos de golf, campos escolares, áreas agrícolas y otros hábitats modificados por humanos. Incluso en su área de distribución nativa, se encuentran en mayores densidades en áreas modificadas por humanos que en su hábitat natural. Su afinidad por el hábitat alterado, la tolerancia a una amplia gama de condiciones y la capacidad de adaptarse a nuevas condiciones son factores importantes que permiten una colonización exitosa en muchos lugares diferentes. Por ejemplo, los sapos de caña prosperan en cuerpos de agua ácida y salina. En Australia, los sapos de caña se han adaptado a los desiertos, y los sapos al frente de la invasión desarrollaron extremidades más largas y la tendencia a moverse en líneas más rectas que los sapos en lugares donde se han establecido por más tiempo. Extremidades más largas y moverse en línea recta acelera el proceso de colonización de otras áreas en Australia.

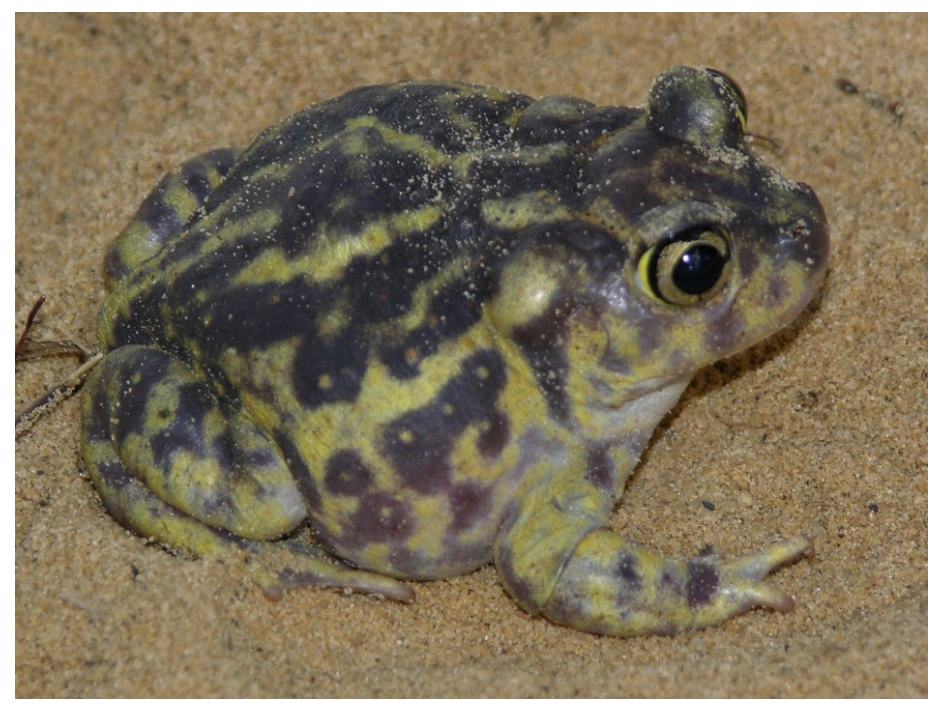

Figure 6. Los sapos orientales de pie de palas nativos del este de Florida generalmente tienen menos de 2 pulgadas de largo y pueden tener numerosas marcas amarillas.

Credits: Steve A. Johnson, UF/IFAS

Como la mayoría de los sapos, los sapos de caña generalmente se encuentran en hábitats terrestres en áreas con fuentes de agua permanente cerca. Para reproducirse, los machos cantan para atraer a las hembras a una variedad de cuerpos de agua, incluidas charcos temporales, estanques y canales, aunque evitan las corrientes rápidas. El canto es un trino persistente, grave y agudo, que puede escuchar aquí: http://www.wec.ufl.edu/extension/wildlife_info/frogstoads/ wav/giant_taod.wav. Los cantos pueden ocurrir durante todo el año en el sur de Florida, pero se limitan de enero a septiembre más al norte del estado. Los sapos de caña son criadores increíblemente prolíficos, las hembras depositan entre 8,000 y 30,000 huevos en cadenas largas y gelatinosas una o dos veces al año. La reproducción generalmente ocurre después de lluvias fuertes. Los renacuajos eclosionan en aproximadamente 48 horas y, dependiendo de la temperatura y la disponibilidad de recursos, requieren aproximadamente dos semanas o dos meses para convertirse en un sapo. El sapo de caña puede crecer mucho más que cualquiera de nuestras ranas y sapos nativos, pero los sapos de caña recién transformados de renacuajos a sapos tienen menos de media pulgada de largo, apenas el tamaño de una pasa, lo que hace difícil distinguirlos de las otras especies de sapos que se encuentran en Florida.

Los sapos de caña adultos son nocturnos, permanecen ocultos durante el día y emergen después del atardecer para buscar comida. Sin embargo, los renacuajos y los sapos más jóvenes son más activos durante el día. Estudios científicos sugieren que esta diferencia en los períodos de actividad ayuda a los sapos pequeños evitar el canibalismo por parte de los sapos de caña más grandes.

El sapo de caña es una especie generalista, come casi cualquier cosa que pueda caber en su boca. Los escarabajos y las hormigas constituyen la mayor parte de su dieta, pero también consumen otros insectos y arañas, carroña, pequeños vertebrados, basura y miembros más pequeños de su propia especie. La comida para perros y gatos que se deja afuera para las mascotas atrae a los sapos de caña y les proporciona una comida fácil.

\section{Impactos en humanos y mascotas}

Los sapos de caña prefieren hábitats creados y habitados por humanos. Las mayores densidades de sapos de caña, así como los individuos más grandes, se encuentran en áreas perturbadas. Son particularmente comunes en patios, campos de golf y campos escolares. Comen casi cualquier cosa de tamaño apropiado y a menudo se sienten atraídos por la comida para mascotas que se deja afuera. Desafortunadamente, los perros y gatos que atacan o intentan comer este sapo altamente tóxico se pueden envenenar. Los perros son considerablemente más propensos que los gatos a intentar comer sapos de caña, especialmente perros terrier, a los cuales les gusta atacar animales pequeños. La gravedad de los síntomas varía según la cantidad de toxina ingerida y el tamaño del animal que atacó al sapo. Los signos de intoxicación por sapo de caña en las mascotas 
incluyen encías inusualmente rosas o rojas, babeo o espuma en la boca, arañazos en la boca, convulsiones y, a veces, paro cardíaco. Los informes de mascotas, particularmente perros, que enferman o mueren después de intentar comer sapos de caña no son infrecuentes en el sur de Florida. Si sospecha que su mascota ha comido o atacado a un sapo de caña, límpielo bien con un trapo húmedo. ¡Y llame inmediatamente a su veterinario!

Los impactos negativos adicionales de los sapos de caña incluyen la toxicidad en peces ornamentales en estanques al aire libre y los impactos potencialmente directos sobre el bienestar humano. Los huevos de sapo de caña y los renacuajos, que son tóxicos, han sido implicado en la muerte de peces koi en estanques ornamentales. Además, la secreción de las glándulas parotoides del sapo de caña es muy irritante para los ojos humanos y en heridas en la piel. Por lo tanto, recomendamos que los sapos de caña se manipulen con guantes y se debe tener mucho cuidado para evitar que las toxinas entren en los ojos: lávese bien las manos después de manipular un sapo de caña. Los sapos de caña afectan negativamente la calidad de vida humana con su canto fuerte. Densas poblaciones de sapos de caña cantando en áreas residenciales durante la noche bajo condiciones adecuadas pueden causar perturbaciones del sueño.

\section{Impactos en el medio ambiente}

Hay pocos registros de sapos de caña en ecosistemas naturales en Florida, aunque se han detectado los cantos de sapos de caña en los Parques Nacionales de Biscayne y Everglades. El bajo número de registros en áreas naturales sugiere que es poco probable que tengan un gran efecto en los hábitats menos perturbados de Florida. Sin embargo, las especies nativas que habitan áreas más urbanas pueden ser vulnerables a los sapos tóxicos. Los sapos de caña son depredadores voraces que podrían reducir la disponibilidad de presas para especies nativas en áreas suburbanas donde las densidades de sapos de caña son mayores. Ya sea debido a la competencia por la comida, la depredación directa u otro factor, las personas han notado que los sapos nativos del sur desaparecen o se vuelven poco comunes en sus vecindarios cuando llegan los sapos de caña.

Aunque su dieta está dominada por artrópodos, también se sabe que los sapos de caña comen varias especies de pequeños vertebrados, incluidas aves nativas, serpientes y pequeños mamíferos. La toxicidad del sapo de caña puede poner en riesgo a las especies nativas, en particular a los depredadores. Algunos animales salvajes nativos tienen adaptaciones que les permiten consumir un animal tan tóxico como el sapo de caña, pero otros son vulnerables a su veneno. Un estudio encontró que algunas ranas nativas pueden comer huevos de sapo de caña, pero otras especies eran susceptibles a su veneno. En Florida no hemos visto las grandes muertes de depredadores como las que se han documentado en Australia, pero se necesita investigación adicional para comprender cómo los sapos de caña afectan a las especies nativas de Florida.

Mire este video para obtener más información sobre los sapos de caña, también conocido como bufo en Florida y cómo tratar a una mascota familiar que tiene un encuentro con un sapo de caña: https://www.youtube.com/ watch? $\mathrm{v}=\mathrm{Cg} 1 \mathrm{HU}$ uxM7Zc.

\section{Lo que usted puede hacer}

Hay varias cosas que usted puede hacer para reducir los impactos de los sapos de caña en Florida y proteger a sus mascotas. Capture a mano y sacrifique humanitariamente a los sapos de caña alrededor de su jardín aplicando generosamente un ungüento o un aerosol de benzocaína o lidocaína en la espalda o el vientre del sapo, luego congélelo durante la noche. Varios productos que contienen benzocaína o lidocaína están disponibles en la mayoría de las farmacias y supermercados sin necesidad de recetas médicas. Algunos ejemplos son productos para tratar dolores de dientes e irritaciones de la piel, como quemaduras solares. No tiene que utilizar el tratamiento de benzocaína, usted puede simplemente poner el sapo de caña en el refrigerador durante unas horas para anestesiarlo antes de transferirlo a un congelador durante 24 horas para garantizar una muerte humanitaria. Aunque el método de enfriar y congelar aún no ha sido aprobado por la Asociación Americana de Medicina Veterinaria como método para sacrificar anfibios como los sapos, un estudio realizado con sapos de caña en Australia encontró que es completamente humanitario. Use guantes de goma o una bolsa de plástico como un guante para capturar sapos a mano. Aunque un sapo no puede arrojarle toxinas, puede exudar toxinas e irritar la piel y los ojos si no se manipula con cuidado. Agarra a los sapos de manera segura: sin dudas y con determinación. Una forma efectiva de sostener un sapo capturado es sujetándolo por la cintura con el pulgar y el índice. Una vez en la mano, gire la bolsa de plástico al revés y átela para contener el sapo antes de ponerla en el refrigerador y luego transferirla al congelador. Debido a que los sapos de caña son principalmente nocturnos, al anochecer es un buen momento para buscar en su propiedad sapos que estén fuera de casa. El uso de una linterna lo ayudará a encontrarlos más fácilmente. 
¡Asegúrese de haber identificado correctamente al sapo como un sapo invasivo antes de sacrificarlo! Revise la información presentada anteriormente en este documento para obtener consejos de identificación. Si todavía no está seguro si tiene un sapo de caña o un sapo nativo, envíe un correo electrónico al Dr. Steve Johnson a tadpole@ufl. edu con una foto del sapo. Él hará todo lo posible para responder a su mensaje. Los estatutos del estado de Florida prohíben la liberación de cualquier animal no nativo en Florida sin un permiso, por lo que es ilegal capturar y luego liberar un sapo de caña. En otras palabras, capturar y luego liberar sapos de caña en otros lugares no es una solución. Tienen la capacidad de regresar a donde fueron capturados originalmente, por lo que llevarlos a otro lugar y liberarlos es ineficaz, lo más probable es que regresen a "casa". Si no regresan, pueden terminar siendo un problema para otra persona o crear una nueva población en un área previamente no invadida.

Para evitar atraer insectos que atraen a los sapos de caña, asegúrese de apagar las luces exteriores alrededor de su hogar. Si apagar una luz no es una opción, use luces de movimiento o reemplace las bombillas que atraen insectos que emiten luz blanca con bombillas conocidas como "luces de insectos", así usted puede reducir la cantidad de insectos alrededor de su hogar por la noche. Además, no deje afuera tazones de comida para mascotas por la noche; los sapos de caña pueden aprender que hay una comida gratis en la puerta de atrás. Los sapos también pueden remojarse en el tazón de agua de una mascota, especialmente durante períodos secos prolongados. Eliminar las fuentes de agua / humedad en su propiedad ayudará a que su jardín sea menos atractivo para los sapos de caña y los mosquitos. Retire los huevos de sapo de caña (colocados en forma de cadenas largas gelatinosas) y los renacuajos (de color negro pequeño y sólido) de los estanques ornamentales o piscinas que no estén bien cloradas. Otra forma de hacer que su jardín sea menos adecuado para los sapos de caña es quitar escombros donde los sapos pueden buscar refugio durante el día. Bloquee el acceso a las pilas de compost y otros escondites, como debajo de las unidades de aire acondicionado o cubiertas y alrededor de los bordes de los edificios.

Para evitar consecuencias potencialmente mortales, no deje a los perros desatendidos al aire libre al amanecer, al anochecer o en la noche cuando los sapos de caña están más activos. Cuando pasee a las mascotas por la tarde o por la noche en áreas donde los sapos de caña son frecuentes, asegúrese de mantener a su perro con una correa corta. Los perros con un fuerte impulso de cazar pueden atacar a un sapo. Si no puede o no desea eliminar los sapos de caña de su propiedad, pero quiere que se vayan, es posible contratar a una persona que atrapa y remueve animales no deseados. Sin embargo, no trabajan de forma gratuita y sus tarifas por la extracción de animales pueden ser costosas, así que obtenga detalles de costos antes de contratar a alguien. Para buscar un cazador de fauna salvaje molesta o no deseada en su área, visite: https://publictemp.myfwc.com/HGM/NWT/ NWTSearch.aspx.

Mire este video para aprender cómo capturar y sacrificar humanitariamente a los sapos de caña invasores en su propiedad: https://www.youtube.com/ watch?v=JCZlSVbOkWU.

\section{Como reportar sapos de caña}

Si encuentra un sapo de caña dentro del área donde se sabe que habitan (Figura 1), informe de su avistamiento en el Sistema de Mapeo de Detección Temprana y Distribución (EDDMapS). Necesitará una imagen digital del sapo y su ubicación específica. La primera vez que reporte un sapo en EDDMapS, se le pedirá que cree una cuenta, esto solo tomara unos minutos. Puede acceder a EDDMapS en https://www.eddmaps.org/report/ y descargar la aplicación "IveGot1" para su teléfono inteligente para que sea más conveniente informar sobre avistamientos de sapos de caña.

Si encuentra un sapo de caña fuera de su rango actual (Figura 1), comuníquese con el Dr. Steve Johnson de la Universidad de Florida a tadpole@ufl.edu. Incluya una foto del sapo, así como el condado y la dirección donde lo encontró. Una vez que haya informado su observación al Dr. Johnson, regístrela en el sistema EDDMapS como se describió anteriormente.

\section{Fuentes Adicionales de Información}

Dr. Steve Johnson's UF Wildlife website has an online guide to Florida's frogs (click link in left column). Be sure to check out the species accounts for cane toads and southern toads. http://ufwildlife.ifas.ufl.edu/index.shtml

The Florida Fish and Wildlife Conservation Commission maintains a page devoted to cane toads that you can find here: http://myfwc.com/wildlifehabitats/nonnatives/ amphibians/giant-toad/ 
National Geographic has a site with cane toad images and a brief summary about their invasion of Australia. http:// animals.nationalgeographic.com/animals/amphibians/ cane-toad/

For more information about cane toads in Australia, please visit the Australian Museum's web page https://australianmuseum.net.au/cane-toad, or Dr. Rick Shine's website http://www.canetoadsinoz.com/ 